\title{
Elasticidades de la demanda mundial de lácteos argentinos y su posicionamiento competitivo
}

Recibido: diciembre 2, 2016 - Aceptado: mayo 23, 2017

Doi: http://dx.doi.org/10.12804/revistas.urosario.edu.co/economia/a.6457

\section{Rodrigo García Arancibia*}

\section{Resumen}

El objetivo del presente trabajo es obtener funciones de demanda internacional de los principales lácteos exportados por la Argentina vis a vis con sus competidores. Con base en el enfoque teórico de Armington, se presenta un modelo para estudiar la demanda de los principales países importadores de leche en polvo y quesos argentinos en función del precio relativo. Se asume, en primer lugar, las preferencias homotéticas, y se propone, luego, una extensión no homotética. Para su implementación empírica, se propone una especificación econométrica para datos de panel. El modelo es estimado utilizando modelos de efectos fijos (filtrado) y efectos aleatorios, con una estructura AR(1) para los errores. De los parámetros estimados se obtiene la elasticidad de sustitución, la elasticidad-precio propia y cruzada en los mercados mundiales relevantes para la Argentina. Tales medidas constituyen una información valiosa para el conocimiento de la respuesta de los compradores mundiales ante diferentes medidas de política o shocks exógenos que pudieran alterar los precios relativos pagados por los importadores, como así también para analizar el posicionamiento de la Argentina en los mercados internacionales.

Palabras clave: modelo de Armington, elasticidad de sustitución constante, elasticidad, precio, efectos fijos, efectos aleatorios.

Clasicación JEL: F14, L66, Q11, Q17

Trabajo desarrollado en el marco del proyecto PICт 2132 de la Agencia Nacional de Promoción Científica y Tecnológica (ANPCyT). Este trabajo forma parte de un capítulo de mi tesis doctoral, y algunos de los resultados fueron presentados en la Reunión Anual de la AAEA, realizada en Tandil-Buenos Aires en octubre de 2015.

* Instituto de Economía Aplicada Litoral (IECAL-FCE-UnL) \& CONICET, Santa Fe, Argentina. Correspondencia. Correo electrónico: rgarcia@fce.unl.edu.ar

Cómo citar este artículo: García A. R. (2017). Elasticidades de la demanda mundial de lácteos argentinos y su posicionamiento competitivo. Revista de Economía del Rosario, 20(2), 275-306. Doi: http://dx.doi.org/10.12804/revistas.urosario.edu.co/economia/a.6457 


\title{
World Demand Elasticities of Argentine Dairy Products and their Competitiveness
}

\begin{abstract}
The aim of this paper is to determine world demand for the main dairy products exported by Argentina vis-à-vis its competitors. Based on the Armington approach, a theoretical model is presented to study the import demand of major importing countries of Argentine milk powder and cheeses as a function of its relative price, extending the traditional homothetic CES specification to a non-homothetic version. For empirical implementation, an econometric specification for panel data is proposed. The model is estimated using filtered fixed effects and random effects models, with an AR(1) structure for idiosyncratic errors. Within the parameters of demand estimates, the elasticity of substitution is estimated as are own-price and cross-price elasticities. Such measures provide valuable information for understanding the response of the global demand to different policy measures or exogenous shocks that could alter the relative Argentine prices paid by importers, and therefore analyze the competitive position of Argentina in some international markets.
\end{abstract}

Keywords: Armington model, constant elasticity of substitution, price-elasticity, fixedeffects, random-effects.

JEL Classification: F14, L66, Q11, Q17

\section{Elasticidades da demanda mundial de lácteos argentinos e seu posicionamento competitivo}

\begin{abstract}
Resumo
O objetivo do presente trabalho és obter funções de demanda internacional dos principais lácteos exportados pela Argentina vis-à-vis seus competidores. Com base no enfoque teórico de Armington, se apresenta um modelo para estudar a demanda dos principais países importadores de leite em pó e queijos argentinos em função do preço relativo, assumindo em primeiro lugar preferencias homotéticas, propondo depois uma extensão não-homotética. Para a sua implementação empírica, propõe-se uma especificação econométrica para dados de painel. O modelo é estimado utilizando modelos de efeitos fixos e efeitos aleatórios, com uma estrutura AR(1) para os erros. Dos parâmetros estimados obtém-se a elasticidade de substituição, a elasticidade-preço própria e cruzada nos mercados mundiais relevantes para a Argentina. Tais medidas constituem uma informação valiosa para o conhecimento da resposta dos compradores mundiais ante diferentes medidas de política ou choques exógenos que puderam alterar os preços relativos pagados pelos importadores, como também assim para analisar o posicionamento da Argentina nos mercados internacionais.
\end{abstract}

Palavras-chave: modelo de Armington, elasticidade de substituição constante, elasticidadepreço, efeitos fixos, efeitos aleatórios.

Classificação JEL: F14, L66, Q11, Q17 


\section{Introducción}

A pesar de que la oferta láctea de la Argentina tiene como destino predominante el mercado doméstico —se exporta aproximadamente solo el $20 \%$ de los litros industrializados-, la demanda externa tiene una gran relevancia para el sector lácteo en su conjunto. Por ello, cumple un rol crucial en la determinación de precios de la cadena y en la competitividad sectorial (Rossini et al., 2013; Vicentin Masaro et al., 2013).

La leche en polvo constituye la principal commodity láctea, lo que representa en promedio más del $70 \%$ del valor total de las exportaciones de los tres principales productos lácteos exportados (leche en polvo, manteca y quesos). En particular, la leche en polvo entera (LPE) supera el $60 \%$ de estas exportaciones, con un crecimiento casi continuo de los valores y volúmenes exportados, lo que muestra un estancamiento en los últimos años con precios internacionales menos favorables (Depetris et al., 2009). Los principales mercados de la LPE han sido históricamente Argelia y Brasil, posteriormente se incorporó Venezuela como principal comprador. En el periodo 2003-2015, estos tres destinos concentraron en promedio más del $66 \%$ de las exportaciones totales de LP. Sin embargo, también existen otros destinos relevantes, en los cuales la Argentina participa de forma continuada y con una significativa participación en el mercado, como Chile, Senegal, Nigeria, Cuba y República Dominicana, entre otros; y, más recientemente, China y otros países del sudeste asiático.

Por su parte, las exportaciones de quesos experimentaron un gran crecimiento en los últimos 15 años, lo que fue acompañado por una diversificación de los tipos de quesos comercializados, con una fuerte expansión de la Mozzarela, ganó participación en destinos no tradicionales como Rusia, Japón, Corea del Sur y Taiwan, entre otros (Depetris Guiguet et al., 2011, 2012).

En los últimos 15 años se observó un cambio de escenario para el sector, con un mayor impulso sobre las exportaciones que se dio en el periodo posdevaluatorio de 2001, seguido por una mayor intervención gubernamental sobre el comercio exterior e importantes shocks en los precios internacionales. No obstante, la industria láctea mostró capacidad de adaptarse al mercado mundial, diversificando su oferta e incrementando su participación en mercados no convencionales. Dados los esfuerzos que implican para los exportadores el mantener y ganar posicionamiento en los mercados internacionales, resulta pertinente conocer la forma en que los países demandantes responden ante diferentes cambios que terminan expresándose en los precios relativos de los bienes importados. Tal conocimiento contribuye al desarrollo de estrategias competitivas de las propias firmas y del sector gubernamental en pos de fortalecer el desempeño externo sectorial. 
Sobre el análisis económico del sector lácteo argentino existe una vasta literatura donde se estudia la evolución de su desempeño competitivo, con una descripción cabal de la demanda internacional como factor clave de la competitividad (e.g. Depetris Guiguet et al., 2009, 2011, 2012; entre otros). Por otra parte, una serie de trabajos proponen modelos explicativos para analizar la oferta exportable de la industria a los mercados internacionales, pero sin abordar cuestiones específicas del comportamiento de los países demandantes (García Arancibia et al., 2013; Depetris Guiguet et al., 2013; Coronel et al., 2014). Por el lado de la demanda de productos lácteos, las investigaciones académicas se han focalizado en la demanda interna (e.g. Lema et al., 2005; Rossini et al., 2008). Sin embargo, no se encuentran estudios que estimen el comportamiento de los compradores mundiales de lácteos argentinos ante variaciones en los precios relativos y demás variables que afectan al comercio, lo que resulta de gran importancia para un análisis completo de la demanda, y con ello de las posibilidades competitivas del sector. Por esto, el presente trabajo busca contribuir, en tal sentido, al proponer un modelo simple de estimar, en cuanto al método y al uso de datos, del cual se obtienen estimaciones de elasticidades de demanda de los principales países importadores.

Para el análisis de la demanda de importaciones, la mayoría las investigaciones adoptan la hipótesis propuesta por Armington (1969) de diferenciación de bienes importados según el país de origen. El modelo original de Armington propone la utilización de preferencias de elasticidad de sustitución constante (preferencias CES) para los países importadores. Esta utilidad CES no solo fue adoptada en numerosas aplicaciones empíricas para la estimación de demandas de importaciones (e.g. Ito et al., 1990; Yang \& Koo, 1993; Feenstra, 1994; Broda \& Weinstein, 2006; Gohin \& Féménia, 2009, Sauquet et al., 2011; Yilmazkuday, 2015), sino que constituye la especificación más difundida en el marco de los denominados modelos gravitacionales de comercio internacional (Anderson \& van Wincoop, 2003; van Bergeijk \& Brakman, 2010). Sin embargo, el supuesto de preferencias CES puede resultar muy restrictivo para el análisis empírico, al asumir homoteticidad y una elasticidad de sustitución constante y equivalente entre pares de exportadores. Por ello, se han propuesto formas más flexibles y, a su vez, consistentes con la teoría económica, como lo son las especificaciones translog (e.g. Kee et al., Tan 2012) o la adaptación de Yang y Koo (1994) del Sistema Casi Ideal de Demanda (AIDS: Almost Ideal Demand System) de Deaton y Muellbauer (1980) para la demanda de importaciones diferenciada por orígenes (SDAIDS: Source Di erentiated Demand System), muy difundido en aplicaciones empíricas para productos y mercados específicos, con diferentes variantes metodológicas 
(e.g. Ben Kaabia \& Gil, 2007; Ramirez \& Wolf, 2008; Wan et al., 2010; Nzaku et al., 2012; Asche \& Zang, 2013; entre otros).

Si bien, el uso de sistemas de demanda flexibles como el SDAIDS resulta muy atractivo, este se aplica cuando se desea estudiar un país específico como comprador-importador, y cuando la periodicidad de los datos lo permiten por los grados de libertad que suponen tales modelos en términos de parámetros y ecuaciones del sistema. Por ello, si lo que se desea es estudiar la demanda mundial, incluyendo la gran mayoría de países compradores y focalizado en un determinado país exportador (i.e. Argentina), debe seguirse otra estrategia de modelización. A su vez, al considerar un mayor número de importadores, existe una mayor dificultad de contar con datos confiables con periodicidad menor que la anual. Por otro lado, con escenarios tan cambiantes, tampoco es recomendable tomar una extensión temporal demasiado amplia para estimar parámetros estáticos de una función de demanda.

Con base en esto, el presente trabajo propone un modelo estático uniecuacional para el estudio de la demanda mundial de leche en polvo y quesos exportados por la Argentina. Si bien, tal modelo sigue los enfoques más tradicionales, como los de Ito et al. (1990) y Yang y Koo (1993), se plantea una versión para ser utilizada en un marco de datos de panel. Esto permite aprovechar la variabilidad derivada de la dinámica temporal y de la heterogeneidad de los compradores, con una especificación log-lineal simple de estimar y consistente con parámetros estructurales del modelo microfundado original. Por ende, el objetivo principal de este trabajo consiste en conocer las elasticidades de la demanda mundial de los lácteos argentinos. Sumado a esto, como objetivo secundario, se busca difundir un modelo de demanda extendido a preferencias CES no homotéticas, con una especificación admisible para datos de panel, que pueda aplicarse a estudios de demanda de importaciones en otros sectores específicos de interés.

Lo que resta del presente trabajo se estructura de la siguiente manera: En la próxima sección se expone el modelo teórico de demanda mundial a seguir. Después, se presenta la estrategia empírica para estimar tales modelos. Luego, los resultados de las estimaciones son explicados y utilizados para analizar el posicionamiento competitivo de la Argentina en el mercado internacional. Para finalizar, se presentan unas breves conclusiones.

\section{Modelo teórico}

Con el fin de modelar la demanda internacional de lácteos argentinos, se toma como base teórica el marco propuesto por Armington (1969), cuya principal 
hipótesis sostiene que los bienes importados con diferentes orígenes, i.e. exportados por diferentes países o regiones, implican diferentes niveles de utilidad al país importador. Por lo tanto, cuando un país importa un cierto bien del resto del mundo, este ya es diferenciado por el solo hecho de tener un país de origen distinto.

El modelo básico de Armington supone que la decisión de importar que realiza un país determinado puede racionalizarse en dos etapas, basado en la separabilidad débil de la función de utilidad. En una primera etapa, el país decide cuánto importar de un determinado bien, asumiendo que tal decisión es separable de las importaciones de otros bienes. Adicionalmente, la mayor parte de la literatura empírica asume que la demanda de importaciones de un bien puede separarse de la demanda correspondiente a la producción doméstica de dicho bien (e.g. Ben Kaabia \& Gil, 2007; Ramirez \& Wolf, 2008; Gohin \& Féménia, 2009; Asche \& Zang, 2013, entre otros).

En la segunda etapa, el país asignará el gasto en importaciones del bien entre diferentes orígenes de forma tal que minimice dicho gasto para un nivel dado de importaciones. De esto se desprende que el país demandante importará el bien de diferentes países exportadores, dependiendo de las preferencias que tiene por el bien de dicho origen y el precio que paga por este, incluyendo los costos de flete y seguro, dado que se asume la minimización del gasto total del bien importado para una cantidad óptima de dicho bien ${ }^{1}$.

Para modelar la demanda mundial de lácteos argentinos vis a vis con sus competidores, se supone que la Argentina ( $a$ ) tiene un total de $M$ países compradores, y en cada $m(m=1, \ldots, M)$ participan $c_{m}$ competidores de $a$. Luego, se asume que cada país $m$ elige el monto a importar del bien de cada origen ( $a$ y $c_{m}$ ), de forma tal que maximice su utilidad $U_{m}$ sujeto a su restricción presupuestaria (primera etapa). De aquí se obtiene la demanda marshaliana de importación del producto en cuestión por parte del país $m, Q_{m}^{*}$ que tendrá la forma general

$$
Q_{m}^{*}=f_{m}\left(p_{a^{\prime}} p_{c_{m}}, Y_{m}\right), \quad m=1, \ldots, M,
$$

donde $p_{a}$ es el precio de importación del origen $a, p_{c_{m}}$ el precio de los competidores e $Y_{m}$ el ingreso de $m$. De aquí, en la segunda etapa para cada importador $m$ se determinan las cantidades compradas del bien a cada exportador,

1 En el modelo original de Armington, cada origen constituye un 'producto'. Con ello, el bien a importar puede pensarse como un mercado de un cierto grupo de productos, i.e. países de origen. 
vía minimización del gasto total para un nivel dado $Q_{m}^{*}$ de la primera etapa. En particular, de aquí se obtiene la demanda mundial de lácteos argentinos:

$$
Q_{m a}^{*}=\psi_{m a}\left(p_{a^{\prime}} p_{c_{m}}, Q_{m}^{*}\right), \quad m=1, \ldots, M
$$

Los supuestos y las formas funcionales específicas adoptadas para $U_{m^{\prime}}$ $f_{m^{\prime}}$ y con ello para $\psi_{m a}$ determinan un cierta ecuación o sistema de demanda de importaciones a ser estimado.

La especificación que es adoptada en este trabajo se basa en la formulación original de Armington, pero adaptada para el estudio uniecuacional de destinos específicos de las exportaciones argentinas de LPE y quesos. Si bien, esta representación posee rigideces derivadas de la teoría económica subyacente, permite derivar una forma funcional parsimoniosa para su implementación empírica. Tales rigideces pueden resumirse en que: (i) La elasticidad de sustitución es constante independientemente de la participación que tiene cada exportador; y (ii) entre cualquier par de exportadores existe una sola elasticidad de sustitución. Ambos supuestos son racionalizados mediante la forma funcional de Elasticidad de Sustitución Constante (CES). Aquí se presentarán dos especificaciones, una más rígida correspondiente a las preferencias homotéticas CES correspondiente al modelo de Armington tradicional, y una extensión que flexibiliza la restricción de homoteticidad.

\subsection{Especificación CES homotética}

Asumiendo que las preferencias de los $M$ países son CES, luego la demanda $Q^{*}$ puede escribirse de la forma

$$
Q_{m}^{*}=\left[\beta_{m a}^{\left(\frac{\sigma m-1}{\sigma_{m}}\right)}+\sum_{j \neq a} \beta_{m j} q_{m j}^{\left(\frac{\sigma_{m}-1}{\sigma_{m}}\right)}\right]^{-\frac{\sigma_{m}}{1-\sigma_{m}}}, \quad m=1, \ldots, M
$$

con $\sum_{j} \beta_{m j}=1$ siendo $\sigma_{m}$ la elasticidad de sustitución en el mercado $m$ y cada $\beta_{m j}$ un parámetro de preferencia del país $m$ hacia $j$ (con $j=a, c_{m}$ ). Luego, a partir de la minimización del gasto para el nivel dado $Q_{m}^{*}$ se obtiene la demanda que realiza el país $m$ del bien importado en cuestión desde la Argentina ( $a$ ) en el momento t. Especícamente, 


$$
q_{m a t}^{*}=\beta_{m a}^{\sigma_{m}} Q_{m t}^{*}\left(\frac{p_{m a t}}{P_{m t}}\right)^{-\sigma_{m}}, \quad m=1, \ldots, M
$$

siendo $P_{m t}$ un índice de precios del bien en el mercado $m$ en el momento $t$. En términos del gasto, sea $G_{m a t} \equiv p_{m a t} q_{m a t}^{*}, G_{m t} \equiv P_{m t} Q_{m t}^{*}$ y $\omega_{m a t} \equiv G_{m a t} / G_{m t}$, luego la expresión doble-logarítmica de la demanda internacional de lácteos argentinos, en términos de su participación en dichos mercados, vendrá dada por

$$
\ln \omega_{m a t}=\sigma_{m} \ln \beta_{m a}+\left(1-\sigma_{m}\right) \ln \left(\frac{p_{m a t}}{P_{m t}}\right), \forall m .
$$

Para un $\sigma_{m}$ común para todo $m$, i.e. $\sigma_{m}=\sigma_{n}=\bar{\sigma}, \forall m, n=1 \ldots, M$ y $m f \neq n$, la especificación (5) representa a la demanda mundial de lácteos argentinos cuya elasticidad-precio propia vendrá dada por

$$
\eta_{a}=-1+(1-\bar{\sigma})-(1-\bar{\sigma}) \omega_{a}
$$

En este caso, $\omega_{a}$ sería la participación media que tiene la Argentina en los mercados mundiales. Considerando que por definición $\sigma_{m} \equiv \partial \ln \left(q_{m a} / Q_{m}\right) / \partial$ $\ln \left(P_{m} / p_{m a}\right)$; luego si cambian los precios de los países competidores $\left(d \ln p_{c} m>0\right)$ y la Argentina mantiene su nivel de precios (i.e. $d \ln p_{m a}=0$ ) y el importador sus importaciones totales del bien (i.e. $d \ln Q_{m}=0$ ), se tiene que

$$
\sigma_{m}=\frac{1}{\omega_{c_{m}}} \frac{\partial \ln q_{m a}}{\partial \ln p_{m c_{m}}}
$$

siendo $\omega_{\mathrm{cm}}$ la participación que tienen los países competidores en el mercado $m$. De esta manera, se obtiene la elasticidad-precio cruzada con los competidores de la Argentina en el mercado internacional, asumiendo $\sigma_{m}=\sigma_{n}=\bar{\sigma}$, $\forall m, n=1 \ldots, M$ :

$$
\eta_{a, c_{m}}=\hat{\sigma} \omega_{c_{m}}
$$

Por lo tanto, la elasticidad-precio cruzada es una proporción de la elasticidad-sustitución constante; y tal proporción es la participación que tienen los países competidores en el mercado $m$. De esta manera, esta elasticidad capta la relevancia que tiene la Argentina en un cierto mercado, escalando de esta 
manera a la elasticidad de sustitución en orden de captar un mayor grado de competencia con los otros exportadores, en aquellos países importadores que poseen una menor participación de mercado.

\subsection{Especificación CES generalizada}

Por la homoteticidad de la especificación CES, la demanda (5) muestra la invarianza de la participación de la Argentina en los mercados mundiales ante cambios en la decisión global de importar lácteos que posee el resto del mundo. Dada la relevancia que tiene conocer cómo impacta el tamaño de los mercados compradores sobre la participación de la Argentina, se presenta una generalización adaptada de la propuesta de Yang y Koo (1993) a los fines de captar diferentes respuestas de la demanda ante cambios en el gasto total. Específicamente, el gasto que realiza $m$ a cada país exportador $j\left(j=a, c_{m}\right)$ puede modelarse de la forma

$$
G_{m j t}=\alpha_{m j} G_{m t}^{\theta_{m j}}, \quad m=1, \ldots, M, \quad j=a, c_{m} .
$$

Escribiendo (4) en términos del gasto, multiplicando ambos miembros por $\frac{p_{m a t}}{P_{m t}}$, se obtiene

$$
G_{m a t}=\beta_{m a}^{\sigma_{m}} G_{m t}\left(\frac{p_{m a t}}{P_{m t}}\right)^{1-\sigma_{m}}
$$

y multiplicando el segundo miembro por $G_{m t} / G_{m t}$ se obtiene

$$
G_{m a t}=\beta_{m a}^{\sigma_{m}} G_{m t}^{2} G_{m t}^{-1}\left(\frac{p_{m a t}}{P_{m t}}\right)^{1-\sigma_{m}} .
$$

De (5) se deduce que $G_{m t}=\alpha_{m a}^{-1 / \theta_{m a}} G_{m a t}^{1 / \theta_{m a}}$, por lo que $G_{m t}^{-1}=\alpha_{m a}^{1 / \theta_{m a}} G_{m a t}^{-1 / \theta_{m a}}$, y sustituyendo en (10) se tiene

$$
G_{m a t}=\beta_{m a}^{\sigma_{m}} \alpha_{m a}^{1 / \theta_{m a}} G_{m a t}^{-1 / \theta_{m a}}\left(\frac{p_{m a t}}{P_{m t}}\right)^{1-\sigma_{m}} G_{m t}^{2}
$$


de esta manera, definiendo $\gamma_{m a} \equiv\left(\frac{\theta_{m a}+1}{\theta_{m a}}\right)$

$$
G_{m a t}^{1 / \gamma_{m a}}=\beta_{m a}^{\sigma_{m}} \alpha_{m a}^{1 / \theta_{m a}}\left(\frac{p_{m a t}}{P_{m t}}\right)^{1-\sigma_{m}} G_{m t}^{2},
$$

lo que se reduce a

$$
G_{m a t}=\beta_{m a}^{\sigma_{m} \gamma_{m a}} \alpha_{m a}^{\gamma_{m a} / \theta_{m a}}\left(\frac{p_{m a t}}{P_{m t}}\right)^{\left(1-\sigma_{m}\right) \gamma_{m a}} G_{m t}^{2 \gamma_{m a}}
$$

De esta manera, en términos de la participación del gasto que tiene $a$ en $m$ en el momento $t$, la demanda será

$$
\omega_{m a t}=\xi_{m a}\left(\frac{p_{m a t}}{P_{m t}}\right)^{\left(1-\sigma_{m}\right) \gamma_{m a}} G_{m t}^{2 \gamma_{m a}-1}
$$

donde $\xi_{m a}=\beta_{m a}^{\sigma_{m} \gamma_{m a}} \alpha_{m a}^{\gamma_{m a}} / \theta_{m a}$. Por lo tanto, tomando logaritmo en ambos miembros, se obtiene la siguiente ecuación de log-lineal de demanda

$$
\ln \omega_{m a t}=\ln \xi_{m a}+\left(1-\sigma_{m}\right) \gamma_{m a} \ln \left(\frac{p_{m a t}}{P_{m t}}\right)+\left(2 \gamma_{m a}-1\right) \ln G_{m t}
$$

A partir de (15), pueden obtenerse las elasticidades precio y gasto correspondientes. En particular, la elasticidad precio-propia vendrá dada por

$$
\eta_{a t}^{p_{m a}}=-1+\left(1-\sigma_{m}\right) \gamma_{m a}\left(1-\omega_{m a t}\right)
$$

Mientras que la elasticidad-gasto será

$$
\eta_{a t}^{G_{m}}=2 \gamma_{m a}
$$

A diferencia de las elasticidades-precio, en este modelo la elasticidad-gasto es $t$-invariante. A pesar de tal restricción, esta generalización del modelo de demanda con utilidad CES permite obtener una medida de cómo afectaría a la demanda de lácteos argentinos un incremento en el poder adquisitivo de los países compradores. Por otra parte, la condición de homogeneidad implicaría restringir $\left(2 \gamma_{m a}-1\right)=0$. Dado que toda función homogénea es homotética, si tal 
condición se cumple la versión generalizada del modelo CES sería equivalente al CES homotético. Por lo tanto, al hacer inferencia sobre el coeficiente $\left(2 \gamma_{m a}-1\right)$ se está contrastando el supuesto de homogeneidad, y con ello enfrentando el CES homotético frente al CES no homotético (Yang \& Koo, 1993).

\section{Metodología}

Para estimar la demanda mundial de quesos argentinos, se construye un panel usando datos anuales de comercio de la base internacional Comtrade (2015). En primer lugar, se analizaron las exportaciones por destinos durante el periodo 1999-2013, para los dos principales productos lácteos exportados por Argentina: leche en polvo entera (LPE) y quesos. A partir de allí, se pudo conocer cuáles son los principales destinos y la continuidad de los envíos de los quesos a tales destinos en dicho periodo, con el fin de formar un panel de datos balanceado. El panel se formó al obtener de cada país de destino $m$, la cantidad y valor por origen, i.e. $Q_{m j t}$ y $G_{m j t}$ respectivamente, para $j=a, c_{m} \mathrm{y} t=1999, \ldots, 2013$. De tales valores fueron computados los precios C.I.F. pagados en el mercado $m$ al exportador $j$ de la forma $p_{m j}=G_{m j j} / Q_{m j j}$. En cada $t$, el gasto y la cantidad total de las importaciones del producto lácteo en cuestión que realiza el importador $m$ es calculado de la forma $Q_{m t} \equiv Q_{a t}+\sum_{i \in c_{m}} Q_{i t}$ y $G_{m t} \equiv G_{a t}+\sum_{i \in c_{m}} G_{i t}$, obteniendo de aquí la participación en el mercado $w_{\text {mat }} \equiv G_{m a t} / G_{m t}$ que constituye la variable respuesta de los modelos de demanda planteados. Como índice de precios en cada mercado $m$ $\left(P_{m}\right)$ para cada producto lácteo considerado (i.e. LPE y quesos), se considera el denominado índice de Stone, que en este caso viene dado por

$$
\ln P_{m t}=\sum_{i=1}^{C_{m}} \omega_{m i t} \ln p_{m i t}
$$

siendo $C_{m}$ el número total de países competidores (exportadores) de quesos en el país $m$.

Los países compradores seleccionados para representar la demanda mundial LPE y quesos son los que se presentan en las tablas 1 y 2 , respectivamente, en las que se detalla la participación media de la Argentina en dichos mercados y los precios promedios recibidos en el periodo 1999-2013. Para el caso de la LPE, se construyó como un solo destino el Sudeste Asiático, uniendo importantes compradores como China, Singapur, Tailandia y Vietnam, 
Tabla 1. Países Importadores de LPE Argentina (Promedios Anuales, periodo 1999-2013)

\begin{tabular}{lccc}
\hline \multicolumn{1}{c}{ Importador $(m)$} & $\bar{w}_{a m}$ & $\bar{p}_{a m}$ & $\bar{G}_{m}$ (C.I.F US\$) \\
\hline Sudeste de Asia & 0,008 & 3,160 & 738000000 \\
Argelia & 0,174 & 3,092 & 474000000 \\
Venezuela & 0,200 & 2,845 & 290000000 \\
Arabia Saudita & 0,006 & 2,703 & 227000000 \\
Brasil & 0,694 & 2,678 & 133000000 \\
Omán & 0,018 & 2,818 & 130000000 \\
México & 0,112 & 2,850 & 82700000 \\
Rep. Dominicana & 0,059 & 2,949 & 79400000 \\
Bangladesh & 0,014 & 2,993 & 71000000 \\
Senegal & 0,148 & 2,710 & 59800000 \\
Líbano & 0,029 & 2,961 & 56000000 \\
Egipto & 0,024 & 2,340 & 48200000 \\
Perú & 0,105 & 2,692 & 27000000 \\
Costa de Mar 1 & 0,105 & 2,686 & 26600000 \\
Níger & 0,401 & 2,714 & 17700000 \\
Chile & 0,634 & 2,685 & 11600000 \\
Bolivia & 0,297 & 2,669 & 4056270 \\
Paraguay & 0,909 & 2,918 & 3981329 \\
Jamaica & 0,581 & 3,480 & 3250650 \\
\hline
\end{tabular}

Fuente: elaboración propia con base en datos de Comtrade (2015).

que si bien representan una importante porción de las exportaciones de LPE, principalmente China en los últimos años, se tiene para cada uno datos interrumpidos. Por ello, se opta por armar un solo bloque que registra compras en todos los años del periodo considerado. Teniendo en cuenta la distancia y los precios implícitos, se observa cierta uniformidad de tales países a fin de ser tomados como uno solo para cubrir todos los años del panel. De esta manera, se obtiene un total de 19 países compradores para representar la demanda mundial de LPE con origen argentino. Por otra parte, en quesos se tiene un total de 13 destinos considerados como los principales países demandantes de la Argentina.

En ambas tablas los datos son presentados en orden decreciente del promedio anual del gasto total realizado por el país $m$ en importaciones de cada lácteo. En general, se observa que en los mercados de mayor tamaño 
Tabla 2. Países importadores de quesos argentinos

\begin{tabular}{lrrr} 
USA & 0,029 & 4,626 & 1000000000 \\
Rusia & 0,018 & 2,733 & 810000000 \\
Japón & 0,010 & 3,817 & 796000000 \\
México & 0,034 & 3,181 & 269000000 \\
Canadá & 0,004 & 5,186 & 195000000 \\
Venezuela & 0,042 & 4,417 & 83300000 \\
Brasil & 0,438 & 3,514 & 61500000 \\
Chile & 0,448 & 3,667 & 32400000 \\
Perú & 0,214 & 3,686 & 9400473 \\
\hline Paraguay & 0,741 & 3,787 & 4952207 \\
Colombia & 0,027 & 5,506 & 3232838 \\
Uruguay & 0,228 & 3,201 & 2167205 \\
Bolivia & 0,353 & 3,287 & 1803209 \\
\hline
\end{tabular}

Fuente: elaboración propia con base en datos de Comtrade (2015).

(i.e. en los países que poseen mayor gasto total en importaciones lácteas), la Argentina participa con una cuota de mercado baja. Esto se da principalmente para los quesos, cuyo mercado internacional se encuentra más atomizado que el de LPE (específicamente, en los destinos aquí considerados), por lo que la Argentina debe enfrentar una mayor competencia en un mercado cuyos productos tienen un mayor grado de diferenciación, respecto a otras commodities lácteas. Incluso para la LPE, hay mercados importadores grandes donde la Argentina posee una importante participación de mercado, como Argelia, Venezuela y Brasil.

Al analizar todos los quesos en su conjunto, se observan diferencias de precios asociadas principalmente con las diferencias en la composición de las importaciones por tipos de quesos. Por ejemplo, los precios de quesos argentinos en el mercado estadounidense son mayores a los de Rusia, porque en el primero se envían principalmente quesos duros, mientras que en Rusia el principal producto enviado es la mozzarella. En este sentido, para filtrar tal efecto es conveniente analizar la demanda por partidas específicas (e.g. Gohin \& Féménia, 2009); sin embargo, en este caso resulta más complicado armar un panel balanceado con una muestra considerable para trabajar empíricamente. Por el contrario, para la LPE los precios son más uniformes, encontrándose algunos precios medios superiores que pueden explicarse 
por el mayor costo de envío como es en el Sudeste Asiático y en Argelia en comparación con los destinos sudamericanos.

Con estos datos de comercio, se ajustan los modelos de demanda correspondientes a la utilidad CES homotética y no homotética descrita en (5) y (15), respectivamente, en sus versiones loglineales en la participación del gasto del importador. Por lo tanto, con el panel formado por los países compradores comentados $(m=1, \ldots, M)$ y el periodo $t=1999, \ldots, 2013$, se tienen las siguientes versiones empíricas de la demanda mundial de quesos argentinos. Bajo la hipótesis de la CES homotética:

$$
\ln \omega_{m a t}=\delta_{m a}+(1-\sigma) \ln \left(\frac{p_{m a t}}{P_{m t}}\right)+u_{m t}
$$

donde $\delta_{m a}=\sigma_{m} \ln \beta_{m a}$ es el efecto específico o individual $t$-invariante y $u_{m a t}$ es un término de perturbación aleatoria. Análogamente, bajo la hipótesis de la CES no homotética, la ecuación empírica de la demanda será:

$$
\ln \omega_{m a t}=\ln \xi_{m a}+(1-\sigma) \gamma_{a} \ln \left(\frac{p_{m t}}{P_{m t}}\right)+\left(2 \gamma_{a}-1\right) \ln G_{m t}+v_{m t},
$$

donde en este caso $\xi_{m a}=\beta_{m a}^{\sigma_{m} \gamma_{m a}} \alpha_{m a}^{\gamma_{m a} / \theta_{m a}}$ es el efecto específico de preferencia en el comercio entre la Argentina y el país $m$ y $v_{m t}$ el término de error. Se debe notar que a los efectos de que los modelos (19) y (20) sean estimables, se supone

$$
\begin{aligned}
\sigma_{m} & =\sigma, \\
\gamma_{m a} & =\gamma_{a} .
\end{aligned}
$$

Los efectos específicos $\delta_{m a} \mathrm{y} \xi_{m a}$ pueden desagregarse en una componente observable y otra no observable. Para la componente observable se consideran algunas variables gravitacionales que pueden ser determinantes de los parámetros de preferencias, como el idioma en común (I), la distancia en millas náuticas (mas allá del efecto sobre costo de trasporte, considerando que $p_{\text {mat }}$ incluye fletes y seguro) y los acuerdos comerciales. Para los acuerdos comerciales se considera al Mercosur ( $M S$ ), al tener los países miembros como compradores relevantes. Dado que Venezuela es incorporado como integrante del bloque a partir de 2006, luego la dummy para Mercosur varía con $t$. Sin embargo, el modelo teórico es flexible para considerar cambios o desplazamientos en el tiempo de alguna componente que sea determinante 
de los parámetros de preferencia $\delta_{m a}$ y $\xi_{m a}$. Por lo tanto, los modelos (19) y (20) pueden escribirse de la forma

$$
\begin{array}{r}
\ln \omega_{m a t}=\delta_{m a}^{*}+\alpha_{i d} I_{m a}++\alpha_{M S} M S_{m t}++\alpha_{d i s t} d i s t_{m a}+\alpha_{\sigma} \ln \left(\frac{p_{m a t}}{P_{m t}}\right)+u_{m t} \\
\ln \omega_{m a t}=\xi_{m a}^{*}+\beta_{i d} I_{m a}++\beta_{M S} M S_{m t}+\beta_{d i s t} d i s t_{m a}+\beta_{\sigma} \ln \left(\frac{p_{m a t}}{P_{m t}}\right)+ \\
+\beta_{G} \ln G_{m t}+v_{m t}
\end{array}
$$

donde, en términos de los parámetros estructurales se tiene que $\alpha_{\sigma} \equiv(1-\sigma)$, $\beta_{\sigma} \equiv(1-\sigma) \gamma_{a}$ y $\beta_{G} \equiv\left(2 \gamma_{a}-1\right)$. Para estimar estos modelos se utilizan en primer lugar técnicas clásicas de datos de panel; a saber el estimador de efectos fijos (EF) y de efectos aleatorios (EA). Al contrastarse la presencia de correlación serial para estos modelos, en ambos se asumirá que los errores siguen un proceso AR(1), i.e.

$$
\begin{gathered}
u_{m t}=\rho^{u} u_{m, t-1}+\varepsilon_{m t}^{u}, \\
v_{m t}=\rho^{v} v_{m, t-1}+\varepsilon_{m t}^{v}
\end{gathered}
$$

Para la estimación de los modelos de EF y EA con AR(1) se emplea el método de Baltagi y Wu (1999), que consiste en estimar en una primera etapa los $\rho^{j}(j=u, v)$ y luego se aplica una transformación sobre los datos con el fin de remover el componente AR(1). Una vez transformados los datos, para el estimador de EF (estimador within), la primera observación de cada panel queda eliminada a fin de remover el efecto fijo, aplicando luego MCO. Para el caso del estimador de efectos aleatorios, sobre estos datos transformados se aplican mínimos cuadrados generalizados (MCGF) ${ }^{2}$.

Dado que el interés de la estimación de las demandas recae principalmente sobre los parámetros del precio, como así también del gasto total en el caso de la CES no homotética, el modelo de EF es preferible ante el riego de estimadores inconsistentes vía MCO o EF en caso de que el efecto individual no explicado esté correlacionado con las covariables del modelo. Sin embargo, el estimador de EF, al usar solo la variabilidad intra (within) es menos eficiente, sumado al hecho de que el estimador within depura la componente fija correspondiente a covariables $t$-invariantes que suelen brindar información de interés sobre las preferencias de los compradores mundiales. Una propuesta

2 Para mayor detalle del método ver Baltagi y Wu (1999), Baltagi (2013) o bien el manual de Stata de xtregar para una revisión rápida de la transformación aplicada para remover el AR(1). 
que contempla las bondades del estimador de EF y a su vez posibilita la estimación de los parámetros correspondientes a variables $t$-invariantes, es el denominado estimador de Descomposición del Vector de Efectos Fijos (DVEF) propuesto por Plümper y Troeger (2007). A pesar de que el DVEF constituye una alternativa atractiva, adoptada por muchos trabajos recientes de datos en panel en general, y para comercio en particular (e.g. Bengoa et al., 2015), existen debates y críticas sobre la originalidad del método respecto al estimador lineal de efectos fijos con variables ficticias, como también sobre la eficiencia y consistencia del estimador (Green, 2011; Breusch et al., 2011; Plümper \& Troeger, 2011).

Recientemente, Pesaran y Zhou (2016) han propuesto un método de efectos fijos que generaliza el DVEF, y del cual se obtienen mejores resultados respecto a la consistencia, insesgadez y eficiencia de los estimadores, con errores estándares robustos por heterocedasticidad y correlación serial. Este método es denominado de Efectos Fijos Filtrado (EFF), el que puede ser computado en 2 etapas. En la etapa 1, para el modelo (19), por ejemplo, se computa el estimador de efectos fijos de $(1-\sigma)$, que se puede denotar por $(1-\hat{\sigma})$, obteniendo el correspondiente residuo

$$
\hat{u}_{m t}=\ln \omega_{m a t}-(1-\hat{\sigma}) \ln \left(p_{m a t} / P_{m t}\right)
$$

En la etapa 2 se calcula, en primer lugar, la media sobre $t$ de tales residuos, i.e. $\overline{\hat{u}}_{m}=\sum_{t} \hat{u}_{m t}$ y se ajusta un modelo lineal (regresión) con intercepto, tomando como respuesta la variable $\overline{\hat{u}}_{m}$ sobre las variables t-invariantes contenidas en $\delta_{m a}$ obteniendo un estimador para tales variables, y el efecto individual del intercepto aquí es computado como la diferencia entre la media de los residuos medios $\overline{\hat{u}}_{m}$ y el valor ajustado de esta última regresión ${ }^{3}$.

\section{Resultados}

\subsection{Coeficientes de la demanda}

En la tabla 3 se presentan los resultados de las estimaciones de la demanda mundial de LPE y quesos, para ambas especificaciones de las preferencias mundiales y diferenciando de acuerdo con el modelo de datos de panel

3 Para mayores detalles de las propiedades de este estimador ver Peasaran y Zhou (2016). 


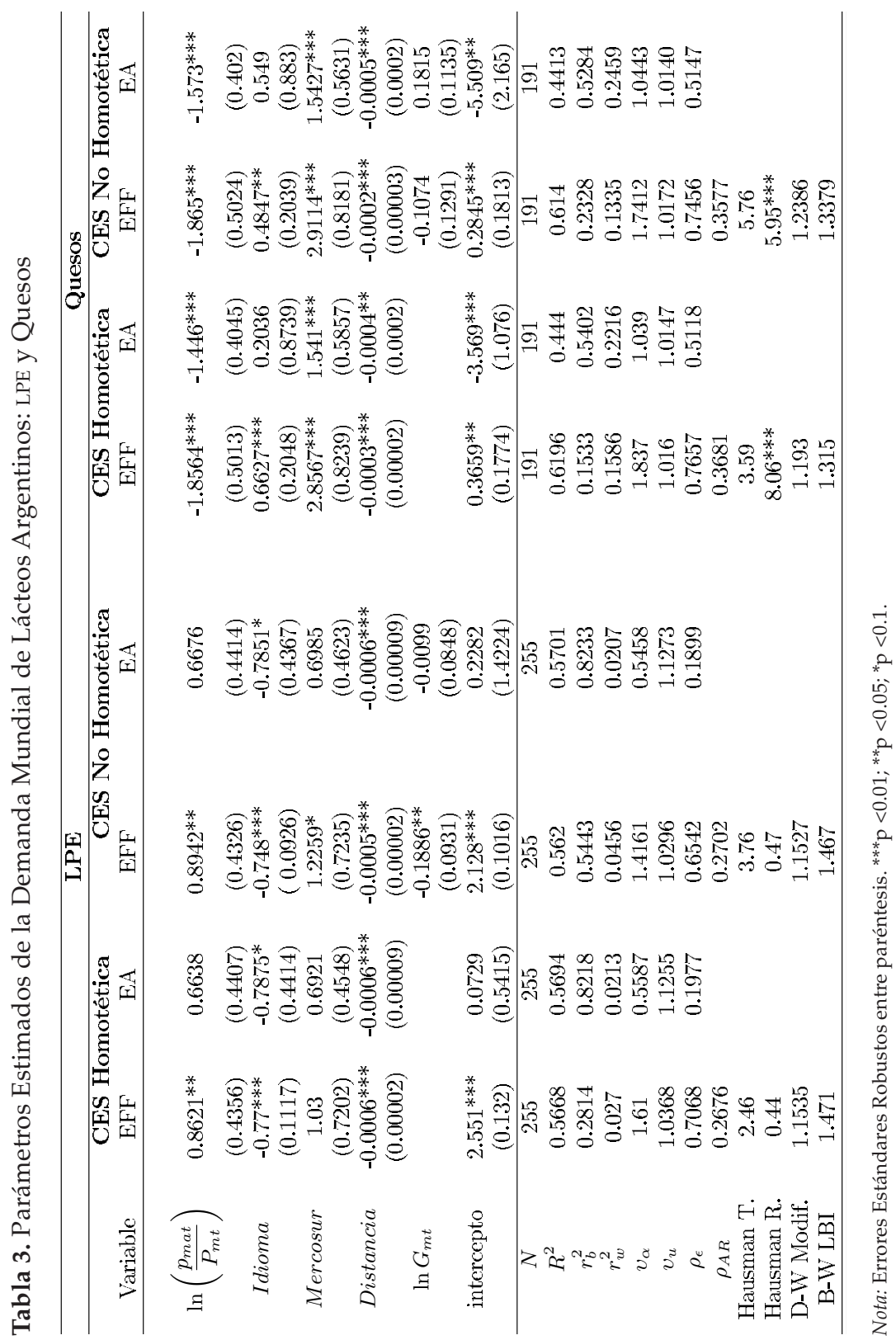


asumido; i.e. efectos fijos (filtrado) estimado vía within y efectos aleatorios vía MCGF, considerando en ambos casos errores idiosincráticos con estructura AR(1). Además de los parámetros de la demanda con sus correspondientes errores estándar, se presentan una serie de medidas y estadísticos adicionales para evaluar la bondad de ajuste y cuestiones de especificación de los modelos. En particular, se muestran diferentes medidas del R-cuadrado, los desvíos estándar del efecto específico no observado y del término de error, los coeficientes de correlación, los estadísticos del test de Hausman (tradicional y en su versión robusta) y dos estadísticos de contraste de la autocorrelación serial; específicamente el estadístico Durbin-Watson modificado propuesto por Bhargava et al. (1983) y el LBI de Baltagi y Wu (1999).

En términos generales, se observan ajustes aceptables en todos los modelos con base en los valores del $R^{2}$ (R-cuadrado global). Con el estimador de EFF, el ajuste mejora significativamente al incluir otras variables respecto al estimador estándar de efectos fijos, teniendo en cuenta que este último ignora la contribución del $\delta_{m a}^{*}$.

Específicamente, para el caso de la LPE, las variables $t$-invariantes contribuyen en aproximadamente un $26 \%$ en la variabilidad explicada del modelo bajo la hipótesis de CES homotética y en un $18 \%$ cuando se asume CES no homotética. En todos los casos, se observa una menor variabilidad explicada within $\left(r_{w}^{2}\right)$ respecto a la between $\left(r_{b}^{2}\right)$, siendo mucho mayor la brecha en el caso de la LPE. Esto sugiere que la variabilidad de demanda mundial de LPE es explicada mayormente por la variabilidad entre los distintos compradores que por variaciones de las importaciones de cada uno de ellos durante el tiempo, reduciendo así la potencialidad que se espera brinde un panel de datos. De todos modos, al tener tan solo dos variables que cambian en $t$, y una de ellas con muy poca variabilidad (Mercosur), resulta lógico obtener un $r_{w}^{2}$ pequeño, dada la dispersión global observada en las cuotas que tiene la Argentina en los distintos mercados internacionales de LPE. Al mismo tiempo, la brecha entre esta medida y el R-cuadrado total muestra que el efecto específico de la heterogeneidad no observada es muy importante. Para los quesos se observa que la variabilidad explicada dentro de cada país durante el tiempo $\left(r_{w}^{2}\right)$ es mayor que en el caso de la LPE, por lo que el estimador de efectos fijos presenta un mejor ajuste. Si bien, entre el valor del $r_{w}^{2}$ y el R-cuadrado total $\left(R^{2}\right)$ hay una menor diferencia, se observa que aún el efecto específico es relevante.

Por otra parte, los valores del D-W modificados y del LBI revelan la presencia de correlación serial para ambos productos y modelos ${ }^{4}$.

4 Para muestras grandes, con valores inferiores a dos se rechaza la hipótesis $\rho_{A R}=0$. 
En los parámetros de demanda estimados se revelan algunas diferencias importantes al comparar efectos fijos frente a efectos aleatorios. Para el caso de la LPE, si se consideran las preferencias CES homotéticas, el coeficiente correspondiente al precio relativo que es mayor en EFF y es estadísticamente significativo, no así bajo EA. En ambos casos, el coeficiente de Mercosur no es significativo a pesar de mostrar el signo esperado. Respecto a las variables $t$-invariantes, hay mayor coincidencia entre EA y EFF, siendo significativas tanto Idioma como Distancia. Para el caso del modelo con preferencias no homotéticas ocurre algo similar en cuanto a la diferencia (y similitud) entre coeficientes. Lo más llamativo en este caso es el coeficiente correspondiente al gasto total en lácteos que realiza el importador $\left(G_{m t}\right)$. Mientras que para el estimador de EA es prácticamente nulo y no significativo, el estimador de EFF muestra un coeficiente negativo y estadísticamente significativo. En términos económicos, esto cobra mucha relevancia, puesto que bajo el estimador de EA, se rechaza la no homoteticidad de las preferencias CES, mientras que con EFF se infiere que ante incrementos en el gasto total lácteo de los importadores, la participación de la Argentina disminuyó, lo que hizo que aumentara, por ende, la de otros exportadores, y colocando así a la LPE argentina en la categoría de bien necesario-inferior en los mercados internacionales respecto a sus competidores.

Para el caso de los quesos se observa que, si bien, los coeficientes del precio relativo difieren entre los estimadores de efectos fijos y aleatorios, en ambos casos son estadísticamente significativos. La variable Mercosur es positiva y significativa, donde el estimador de EFF casi que duplica al de EA. Para la variables $t$-invariantes, la mayor diferencia está en la variable Idioma, siendo solo significativa con el estimador de EFF. La variable Distancia es significativa para ambos estimadores, pero en este caso EA arroja mayores coeficientes. Para la CES no homotética de quesos, el gasto total del país importador muestra no ser estadísticamente significativo, según ambos estimadores. Por lo tanto, para los quesos puede asumirse que bajo el supuesto de utilidad de elasticidad constante (CES) de los importadores, la hipótesis de homoteticidad no se rechaza en términos estadísticos.

Al encontrar tales divergencias entre los estimadores de los coeficientes de demanda, resulta conveniente contrastar la hipótesis nula de que los efectos específicos son aleatorios, distribuidos independientemente de los predictores. Como se observan en los estadísticos de las tablas, para la LPE tanto el

Para muestras pequeñas, los límites (inferior - superior) de los valores críticos específicos según tamaño de $T$ y $N$ se puede ver Bhargava et al. (1982), o Verbeek (2008) para una explicación más coloquial de este con algunos valores críticos. 
test de Hausman tradicional como su versión robusta muestran que no existe evidencia estadística suficiente para rechazar los supuestos del modelo de EA. Si bien, en la literatura económica aplicada suele recomendarse la utilización de efectos fijos, dado que es muy común encontrar argumentos económicos para respaldar la correlación entre el efecto específico no observado y las covariables incluidas, en el presente se tomarán en cuenta ambos estimadores a los fines comparativos; y se verá, asimismo, que las principales elasticidades derivadas de tales modelos suelen converger a valores similares. Para los quesos, si bien el test de Hausman tradicional no rechaza los supuestos del modelo de EA, el Hausman robusto rechaza a un nivel del $1 \%$, por lo que en este caso hay mayor evidencia a favor del uso del estimador de efectos fijos.

Para la LPE, los coeficientes estimados del precio relativo, i.e. $1-\sigma_{m} \mathrm{y}$ $\left(1-\sigma_{m}\right) \gamma_{m a}$ muestran, en general, una baja elasticidad de sustitución en los mercados internacionales donde la Argentina participa con sus exportaciones. Específicamente, los valores estimados de estos coeficientes muestran que un incremento (caída) relativo del precio de LPE argentina estuvo asociado con un aumento (disminución) en su participación de mercado, siendo un resultado no consistente con la teoría de demanda, dado que en general se espera una elasticidad de sustitución mayor a la unidad, o, lo que es equivalente, unos valores de $1-\hat{\sigma}_{m} \mathrm{y}\left(1-\hat{\mathrm{\sigma}}_{m}\right) \hat{\gamma}_{m a}$ negativos (Armington, 1969). Sin embargo, la elasticidad de sustitución, aunque baja, sigue teniendo el signo esperado. Este resultado de baja $\sigma(<1)$ se observa en varios casos de la literatura empírica que utiliza especificaciones CES para el análisis de la demanda de importaciones para diferentes tipos de productos, como en Yang y Koo (1993), Gohin y Féménia (2009) o Sauquet et al. (2011). Esto, como se verá posteriormente, termina reflejándose en bajas elasticidades precio, propias y cruzadas.

Por otro lado, para el mercado internacional de quesos, contrariamente a lo que se observa para la LPE, el coeficiente del precio relativo es negativo y superior a la unidad en valor absoluto, lo que revela una elevada elasticidad de sustitución. En este caso, un incremento relativo del $10 \%$ en el precio de los quesos argentinos reduciría la participación media de la Argentina en el mercado mundial en aproximadamente un $18 \%$, de acuerdo con el estimador de efectos fijos.

Mientras que el signo de la distancia es el esperado (negativo) y en ambos productos es una variable significativa, el idioma en común para el modelo de LPE tiene signo negativo, mientras que en quesos es positivo, y en ambos casos estadísticamente significativo cuando se considera el estimador de efectos fijos. En general, el idioma en común (o las raíces coloniales comunes) funciona como proxy de la distancia cultural entre países, y generalmente está asociada, de forma inversa, con los costos de transacción del comercio 
(Agostino et al., 2007; Fidrmuc, 2009). Por ello, la hipótesis respecto al idioma en común es que este contribuya a facilitar las relaciones comerciales y por ende tenga un impacto positivo sobre la demanda. Sin embargo, para la LPE es negativo. Dado que los países de habla hispana con los que Argentina comercia LPE son, en general, países limítrofes o muy cercanos de Sudamérica, algunos de ellos socios del Mercosur como Paraguay o Venezuela, la variable Distancia ya capta este favoritismo por la cercanía entre estos. Cuando los modelos se ajustan sin la variable Distancia, el idioma en común sigue siendo significativo, pero con el signo positivo esperado. Sin embargo, si se incluye solo Idioma, resulta confuso conocer cuál es el factor que realmente facilita el comercio, es decir, si es el idioma en común, o bien la cercanía entre los países que casualmente hablan el mismo idioma. Entre los países cercanos a la Argentina y que poseen otro idioma se encuentra Brasil, quien constituye uno de los principales países compradores de lácteos argentinos. De hecho, la participación media de Brasil es del 69\%, mientras que la del resto de los países sudamericanos de habla hispana es del $43 \%$. Esto se refuerza más aún si se considera que fuera de Sudamérica solo se encuentran República Dominicana y México como compradores de habla hispana, donde Argentina tiene una participación media (en todo el periodo) del 8,6\%, en contraposición al $13,5 \%$ en países compradores que no pertenecen a Sudamérica y poseen otro idioma. Por lo tanto, el signo negativo del idioma en común queda totalmente justificado en este sentido, siendo consistente con la distribución de la demanda revelada en los mercados internacionales.

\subsection{Análisis de las elasticidades}

De los coeficientes estimados de las funciones de demanda presentados anteriormente, es posible computar las diferentes elasticidades consistentes con los modelos estructurales propuestos. En primer lugar, la elasticidad de sustitución $(\sigma)$ surge directamente del coeficiente del precio relativo. A partir de (6) y (16), se obtienen las elasticidades-precio propias de la demanda de LPE y quesos exportados por la Argentina $\left(\eta_{a}\right)$. Con base en (7), se computa la elasticidad-precio cruzada de la respuesta de demanda de LPE y quesos argentinos respecto del precio de los países exportadores que compiten con la Argentina en los mercados mundiales analizados $\left(\eta_{a, c_{m}}\right)$. Por último, con base en el modelo con CES no homotética, es posible obtener la elasticidadgasto $\left(\eta_{a t}^{G_{m}}\right)$ con base en (17). Puesto que las elasticidades-precio varían de acuerdo con las dos dimensiones del panel (i.e. tiempo y países), para obtener una medida sintética, se calculan en la media (i.e. tomando $\omega_{a}=\sum_{m} \sum_{t} \omega_{m a t}$ ). 
A su vez, a partir del método delta se obtienen los errores estándar correspondientes. Los resultados de estas elasticidades son resumidos en la tabla 4.

Tabla 4. Elasticidades de la demanda mundial de lácteos argentinos

\begin{tabular}{|c|c|c|c|c|}
\hline \multirow{4}{*}{ Elasticidades: } & \multicolumn{2}{|c|}{ CES Homotética } & \multicolumn{2}{|c|}{ CES No Homotética } \\
\hline & EFF & EA & EFF & EA \\
\hline & & & & \\
\hline & & & & \\
\hline Sustitución $(\sigma)$ & $\begin{array}{c}0.1379 \\
(0.4357)\end{array}$ & $\begin{array}{c}0.3362 \\
(0.4407)\end{array}$ & $\begin{array}{c}0.1058 \\
(0.4326)\end{array}$ & $\begin{array}{c}0.3324 \\
(0.4414)\end{array}$ \\
\hline Precio & & & & \\
\hline Propia $\left(\eta_{a}^{p_{m a}}\right)$ & $\begin{array}{c}-0.335 \\
(0.3368)\end{array}$ & $\begin{array}{l}-0.4868 \\
(0.313 \check{)})\end{array}$ & $\begin{array}{l}-0.3087 \\
(0.3407)\end{array}$ & $\begin{array}{c}-0.4839 \\
(0.3413)\end{array}$ \\
\hline Cruzada $\left(\eta_{a}^{p_{c m}}\right)$ & $\begin{array}{c}0.1066 \\
(0.3268)\end{array}$ & $\begin{array}{c}0.2599 \\
(0.3407)\end{array}$ & $\begin{array}{c}0.0818 \\
(0.3344)\end{array}$ & $\begin{array}{c}0.2569 \\
(0.3413)\end{array}$ \\
\hline Gasto $\left(\eta_{a t}^{G_{m}}\right)$ & 1 & 1 & $\begin{array}{c}0.8114 \\
(0.0931)\end{array}$ & $\begin{array}{c}0.9901 \\
(0.0848)\end{array}$ \\
\hline Quesos & & & & \\
\hline Sustitución $(\sigma)$ & $\begin{array}{c}2.6770 \\
(0.4991)\end{array}$ & $\begin{array}{c}2.44 \leftrightharpoons 6 \\
(0.4045)\end{array}$ & $\begin{array}{c}2.8648 \\
(0.5024)\end{array}$ & $\begin{array}{c}2.5732 \\
(0.4020)\end{array}$ \\
\hline Precio & & & & \\
\hline Propia $\left(\eta_{a}^{p_{m a}}\right)$ & $\begin{array}{l}-2.3430 \\
(0.3997)\end{array}$ & $\begin{array}{l}-2.1579 \\
(0.3240)\end{array}$ & $\begin{array}{l}-2.4937 \\
(0.4024)\end{array}$ & $\begin{array}{l}-2.2601 \\
(0.3220)\end{array}$ \\
\hline Cruzada $\left(\eta_{a}^{p_{c_{m}}}\right)$ & $\begin{array}{c}2.1440 \\
(0.3998)\end{array}$ & $\begin{array}{c}1.9589 \\
(0.3240)\end{array}$ & $\begin{array}{c}2.2947 \\
(0.4024)\end{array}$ & $\begin{array}{c}2.0611 \\
(0.3220)\end{array}$ \\
\hline Gasto $\left(\eta_{a t}^{G_{m}}\right)$ & 1 & 1 & $\begin{array}{c}0.8926 \\
(0.1291)\end{array}$ & $\begin{array}{c}1.1815 \\
(0.1136)\end{array}$ \\
\hline
\end{tabular}

Nota: E.E. entre paréntesis. Computados vía método delta.

En primer lugar, como ya fue comentado en la sección anterior, se observa una gran diferencia entre las elasticidades de sustitución de la LPE y de quesos. En el primer caso, son bajas y no significativas, mientras que para los quesos son mucho más altas y significativas. Esto sugiere la existencia de un mayor nivel de competencia en los mercados mundiales de quesos, donde los países demandantes tienen una mayor respuesta ante cambios en los precios relativos. Por el contrario, el mercado mundial de LPE se muestra más invariante ante diferencias en los precios relativos, revelándose por ende una menor presión competitiva.

Esta conclusión se refuerza al observar los valores de las elasticidadesprecio. Mientras que en LPE la demanda mundial es inelástica ante los precios propios (i.e. de Argentina) y de los competidores, la demanda mundial de quesos es elástica, lo que muestra que ante un incremento del $10 \%$ en los precios de los quesos argentinos, la cantidad demandada de estos se reduce en 
promedio en más del 20\%. Del mismo modo, ante un incremento del $10 \%$ en el precio de los competidores, la cantidad demandada de quesos argentinos se incrementaría también en más del $20 \%$. Si bien, las elasticidades-precio propias son mayores a las cruzadas en valor absoluto (lo que se espera desde la teoría microeconómica), la diferencia no es tan sustancial.

Las elasticidades-gasto son cercanas a la unidad, lo que fue previamente deducido a partir de su coeficiente de demanda cercano a cero; se muestra con ello evidencia a favor del supuesto de homoteticidad de las preferencias. A pesar de ello, si se consideran los estimadores de EFF, puede observarse que ante un incremento en el gasto total en lácteos que realizan los importadores, la cantidad demandada hacia los lácteos argentinos se incrementa pero en una proporción un poco menor (19\% para la LPE y 11 \% para quesos). Adicionalmente, se puede ver que las elasticidades-gasto para quesos son mayores que las de LPE, lo que es consistente con el hecho de que los quesos son bienes más de lujo que la LPE y se muestra, incluso, un estimador de la elasticidad mayor que uno bajo el estimador de EA.

Debe tenerse en cuenta que las elasticidades-precio son estimadas tomando los precios pagados por los países consumidores de las importaciones lácteas, i.e. precios C.I.F. $p_{m a}$; y por de nición $p_{m a}=\tau_{m a} \tilde{p}_{m a}$ donde $\tau_{m a}$ representa los costos de fletes, seguros y aranceles, y $\tilde{p}_{m a}$ es el precio de la exportación (F.O.B.) que realiza $a$ al destino $m$. Por lo tanto, si los precios de exportaciones son constantes, las elasticidades-precio pueden reflejar los cambios en la demanda por cambios en los costos efectivos de transporte incluyendo el efecto de la política arancelaria.

Dado que las estimaciones de las elasticidades se obtienen a partir de modelar la esperanza condicionada de la demanda tomando un panel conformado por un periodo histórico (1999-2013) y un conjunto de países compradores, se puede realizar una interpretación económica de estas, teniendo en cuenta tales dimensiones del panel de datos. De esta manera, como en el caso de LPE la variabilidad explicada tiene su origen principalmente en la dispersión temporal, con los valores de la elasticidad-precio propia podría concluirse que para los años en los que los precios medios de la Argentina fueron superiores en un $10 \%$, la cantidad demandada fue en promedio un $4,8 \%$ menor (considerando la elasticidad-precio propia del modelo de EA). Para el caso de los quesos, la variabilidad explicada entre países compradores también es relevante, por lo que los resultados de la elasticidad deben interpretarse teniendo en cuenta ambas fuentes de variabilidad. Específicamente, los resultados muestran que en años y países donde el precio de los quesos argentinos fue un $10 \%$ superior, se demandó en promedio un $20 \%$ menos. Con esto se quiere puntualizar que si bien los valores de las elasticidades 
(evaluados en la media total) permiten caracterizar el comportamiento de la demanda en términos generales, debe tenerse en cuenta que la derivación de tal valor numérico se extrajo de un periodo histórico y de países compradores heterogéneos, para los cuales se tienen elasticidades puntuales específicas.
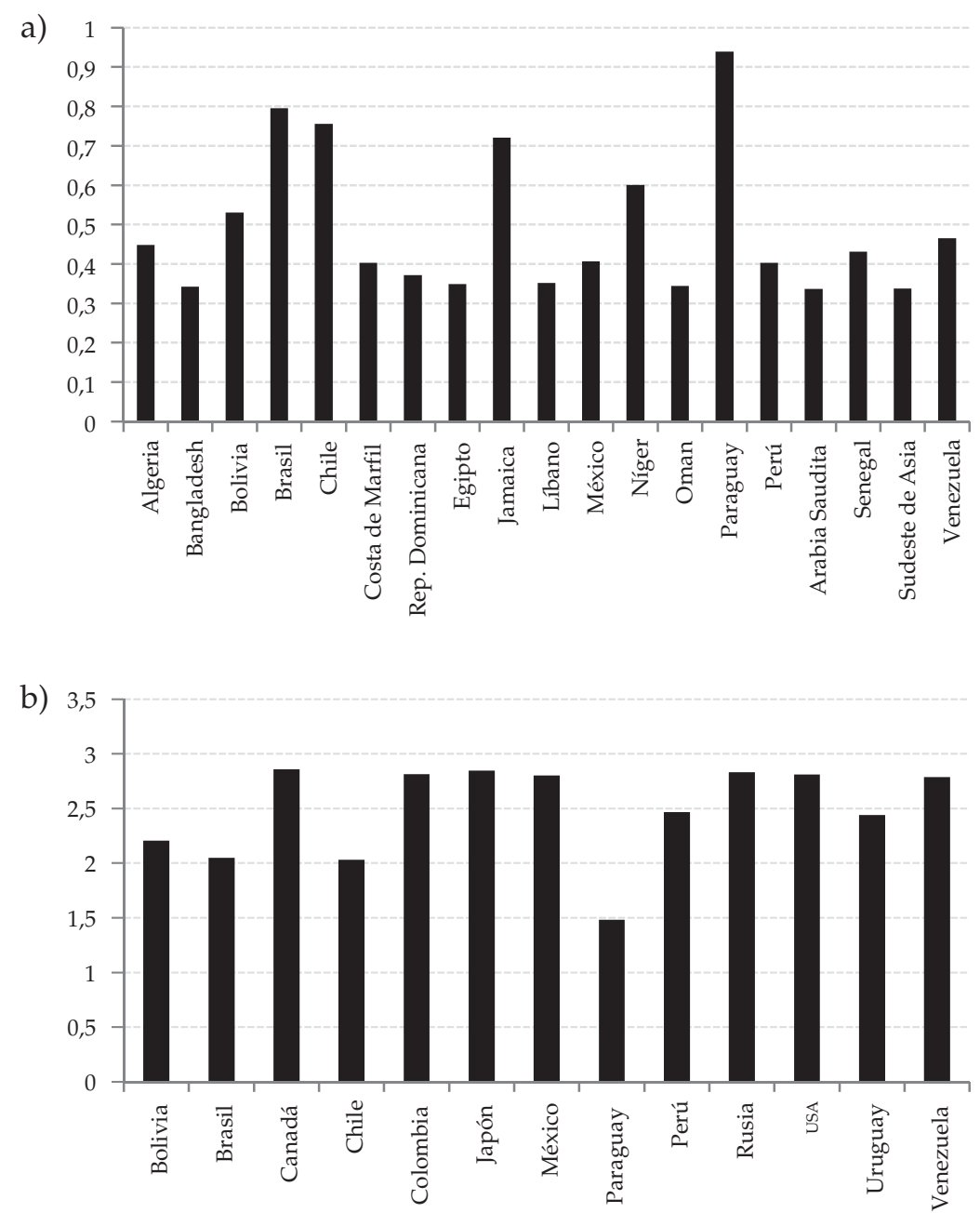

Figura 1: Elasticidad-precio propia en países importadores específicos.

(a) Mercado Mundial de LPE; (b) mercado mundial de quesos

Si bien, para la estimabilidad de las funciones de demandas es necesario asumir una elasticidad de sustitución constante común para cada país de destino, la elasticidad-precio propia es función de la participación que tiene la Argentina en el destino específico. Por ello, para cada país importador y 
en un año específico se tiene un determinado valor de la elasticidad, a pesar de que el componente correspondiente del grado de sustituibilidad entre competidores mundiales se asuma idéntico en cada destino. De (6) se observa que en el caso de baja elasticidad de sustitución (i.e. $(1-\bar{\sigma})>0$ ), como en el mercado mundial de LPE, cuanto mayor sea la participación de la Argentina en cierto mercado $m$, mayor $\left|\eta_{m a}\right|$. Es decir, que la inelasticidad respecto al precio asociada con la baja elasticidad de sustitución se ve contrarrestada cuando la Argentina tiene una mayor cuota de mercado. Por el contrario, en el caso de alta elasticidad de sustitución (i.e. $(1-\bar{\sigma})<0$ ), como en el mercado de los quesos, la mayor participación de la Argentina en un cierto mercado $m$ implica una menor $\left|\eta_{m a}\right|$. Por lo tanto, cuando existe alto grado de sustituibilidad entre los competidores, los mercados compradores son más insensibles a cambios en los precios, si la Argentina tiene un mayor posicionamiento en dicho mercado. Por el contrario, en los mercados con baja participación y demanda más elástica, la reducción porcentual en la cantidad demandada ante un incremento en el precio será mayor.

En las figuras 1 (a)-(b) se exponen las elasticidades-precio propias de la demanda de LPE y quesos argentinos en cada mercado específico $m$.

Para la LPE se observa que en países sudamericanos como Brasil, Chile, Bolivia y Paraguay, donde la Argentina mantiene mayores cuotas de mercado, la elasticidad-precio propia llega a duplicar a la elasticidad promedio. También para países como Jamaica o Nigeria se observan elasticidades superiores. Para el resto de los países, las elasticidades rondan el valor de la media, esto es entre 0,3 y 0,4 en valor absoluto, superando levemente estos valores en mercados más relevantes para las exportaciones argentinas de LPE como Argelia, Senegal y Venezuela.

Por el contrario, en los mercados internacionales de quesos se observa cómo en países donde la Argentina tiene mayor participación, la demanda es más insensible ante cambios en los precios argentinos de importación, mientras que en mercados grandes y más atomizados en las importaciones como Estados Unidos, Mexico, Canada, Japón y Rusia, la demanda por quesos argentinos llega ser casi igual a 3 en valor absoluto.

Haciendo uso de estas elasticidades, es posible construir una medida del grado de competencia, y con ello del posicionamiento competitivo de la Argentina, con base en ponderar la medida de elasticidad por la participación que tiene en el mercado. Específicamente para un destino $m$, se define el siguiente ratio

$$
I C_{m}^{a}=\frac{\omega_{m a}}{\left|\eta_{m a}\right|}
$$


El ratio $I C_{m}^{a}$ constituye una medida aproximada del poder de mercado que revela la Argentina en un cierto país comprador, y es un indicador de competitividad refinado respecto a la simple participación en el mercado mundial, al incorporar la respuesta de la demanda en la fijación de los precios propios. Mas aún, en el marco de la Nueva Organización Industrial Empírica (NOIE), si se considera el problema de decisión de un país exportador respecto a la colocación de su producto en el mercado $m$ en un marco de competencia imperfecta, bajo ciertos supuestos en torno al parámetro de "variaciones conjeturales", I $C_{m}^{a}$ constituye una proxy del denominado índice de Lerner (i.e. $\left[p_{m a}-c^{\prime}\left(q_{m a}\right)\right] / p_{m a}$ siendo $c^{\prime}\left(q_{m a}\right)$ el costo marginal de producción de $\left.q_{m a}\right)$ (Suzuki \& Kaiser, 2006). En el presente trabajo se considera al ratio IC como un índice del posicionamiento competitivo que tiene en los mercados internacionales, pues este es creciente cuando el país tiene una participación alta en un mercado más insensible ante cambios en los precios propios, lo que revela una mayor potencialidad para mantener e incrementar sus ventas en dicho mercado. Los resultados del $I C_{m}^{a}$ se presentan en las figuras 2 (a) y (b).

Para los importadores de LPE se pueden clasificar tres grupos de países respecto al posicionamiento de la Argentina. En primer lugar, en los países de Sudamérica se revela el mayor posicionamiento competitivo. Por lo tanto, si bien la respuesta de la demanda respecto a los precios es mayor (figura 4.2), esto no llega a contrarrestar la importante participación en el mercado que tiene, y con ello el mayor poder de mercado. Con un posicionamiento medio, se encuentran algunos países africanos como Argelia, Niger y Senegal, o bien Venezuela, México y Perú, los que a su vez mostraron menores elasticidadesprecio. Por último, un posicionamiento relativamente débil se observa en mercados más distantes como los de Arabia Saudita, Omán, Líbano, China y el sudeste asiático (agrupados en Sudeste de Asia).

En el comercio de quesos, se observan diferencias más pronunciadas entre países sudamericanos donde se revela un alto posicionamiento competitivo de la Argentina (exceptuando Venezuela y Colombia), y el resto de los países donde el IC es cercano a cero. En este caso, la baja participación en dichos mercados es reforzada por mayores elasticidades-precio, siendo mercados en donde la coexistencia de importantes competidores mundiales y diferentes acuerdos comerciales entre ellos hace más dificultoso el incremento en la participación de la Argentina en la demanda de importación láctea total. 
a)

Participación de mercado/elasticidad

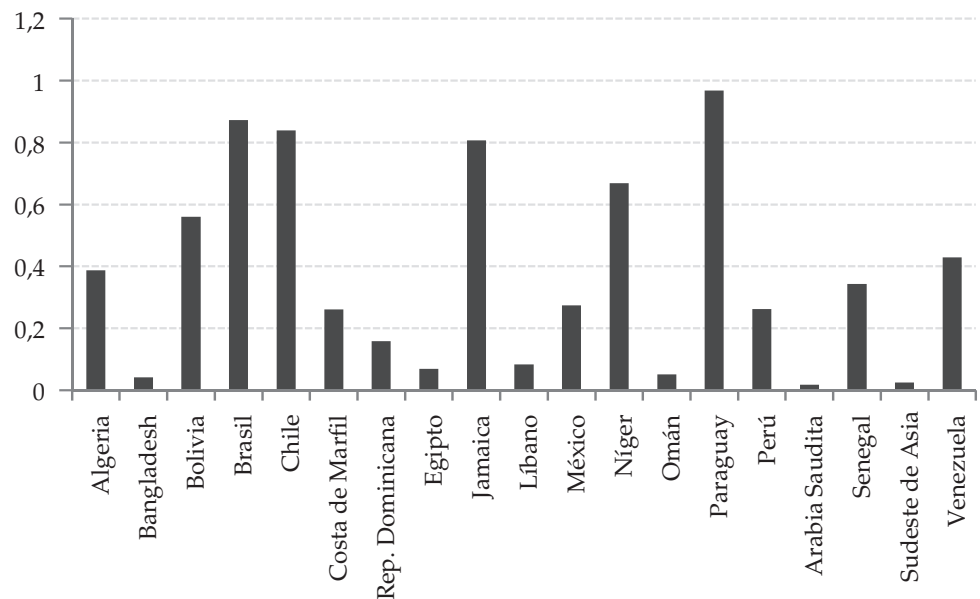

b)

Participación de mercado/elasticidad

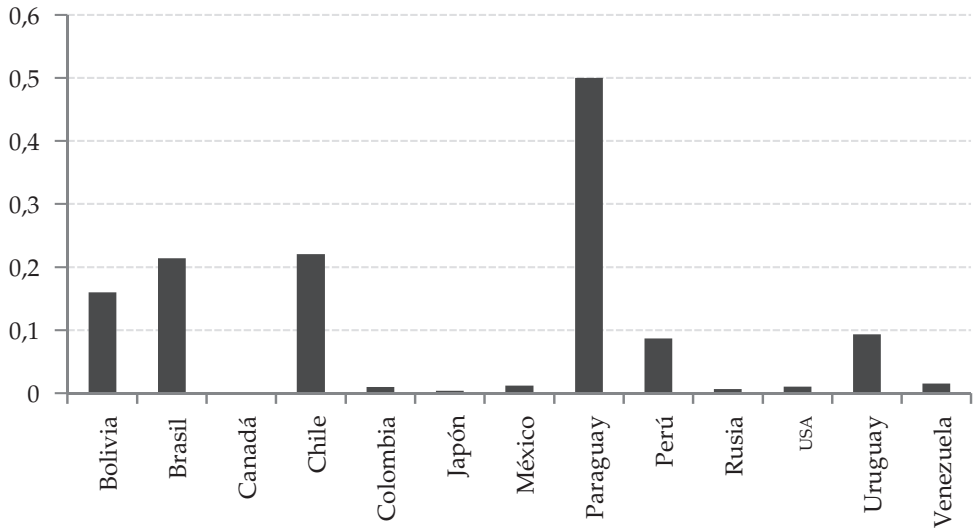

Figura 2. Posicionamiento en los mercados mundiales. Ratio $I C_{m}^{a}$.

\section{Conclusiones}

En el presente trabajo se estudió la demanda mundial de lácteos argentinos tomando como base los principales países compradores en el periodo 19992013. Con un enfoque teórico microfundado con base en la hipótesis de diferenciación de productos por orígenes de Armington, se propuso un modelo en que cada país importador demanda LPE y quesos argentinos en función del precio relativo (de la Argentina frente a sus competidores) y de otras variables gravitacionales que determinan los parámetros de preferencia de la 
función de demanda. Específicamente, se asumió que los países compradores tienen una utilidad de elasticidad de sustitución constante (CES), se propone así una versión homotética y una extensión no homotética de esta. Tal modelo es presentado de forma tal que en su versión empírica queda expresada para trabajar con datos de panel, utilizando datos anuales de fácil accesibilidad.

Para estimar las funciones de demanda se utilizan métodos de efectos fijos filtrado (EFF) y efectos aleatorios (EA), asumiendo un proceso autoregresivo de orden uno en los errores. A partir de los parámetros estimados de las demandas, se computan las diferentes elasticidades asociadas con base en cada ecuación estructural de demanda.

Los resultados de las estimaciones muestran ajustes aceptables para los modelos de demanda propuestos, y en general todas las variables incluidas son estadísticamente significativas, y en particular si se consideran los estimadores de EFF. La excepción se da en la variable correspondiente al gasto total en importaciones para la demanda de quesos, mostrando con ello evidencia a favor de una especificación homotética para dicha demanda. Para la demanda de LPE, el estimador de efectos fijos muestra un coeficiente negativo y significativo para el gasto total, categorizando así a la LPE argentina, como un bien necesario-inferior para la demanda mundial.

Para la distancia, los coeficientes tienen el signo esperado; es decir, que en países más cercanos la participación de la Argentina en los mercados de LPE y quesos importados es mayor. Por otra parte, el hecho de que el importador sea socio del Mercosur tiene un impacto positivo y significativo en la participación de la Argentina en el orden de casi el 3\% para la demanda de quesos, siendo mucho menor para la demanda de LPE, el que es solo significativo en el modelo no homotético de efectos fijos.

El idioma en común muestra un signo contrario al esperado para la demanda mundial de LPE, lo que queda justificado por la distribución que existe entre los distintos países demandantes. Para la demanda de quesos, el idioma en común muestra el signo esperado, i.e. la participación de la Argentina en los mercados internacionales es significativamente mayor cuando el país comprador es de habla hispana.

Del coeficiente del precio relativo se revela que en los mercados de LPE la tasa de sustitución ante cambios en los precios relativos es muy baja, y con ello, la demanda mundial ante cambios en los precios de la LPE argentina es inelástica. Específicamente, la elasticidad-precio propio muestra que para el periodo considerado, un incremento del $10 \%$ en el precio de la LPE argentina implicó una reducción de aproximadamente $4 \%$ en la cantidad demandada de esta. Por el contrario, para el mercado internacional de quesos importados existe un elevado grado de sustitución ante cambios en los precios relativos, 
revelándose una demanda mundial bastante elástica. Con una elasticidadprecio propia superior a 2 , los resultados muestran que durante el periodo 1999-2013, precios de los quesos argentinos superiores en un $10 \%$ significaron en promedio una reducción mayor al $20 \%$ en la cantidad demandada. También para la elasticidad precio-cruzada se observan valores cercanos a 2, levemente inferiores a las elasticidades-precio propias.

Del indicador construido como el cociente entre la participación de mercado de la Argentina y el valor absoluto de la elasticidad-precio estimada, se revela un alto desempeño competitivo de la Argentina en los países sudamericanos. Para los mercados africanos de LPE, que constituyen importantes compradores de la Argentina, se revela un posicionamiento competitivo medio en comparación con otros destinos como Arabia Saudita, Omán, China y el Sudeste asiático, donde se revela el menor posicionamiento competitivo. Para los quesos, más allá de los destinos sudamericanos, en el resto de los mercados el indicador propuesto es cercano a cero, lo que es explicado por la baja participación que tiene la Argentina en estos junto con la elevada elasticidad-precio propio de la demanda.

Por último, cabe destacar que, si bien, el trabajo tuvo como objetivo principal conocer las elasticidades de la demanda mundial de los principales lácteos argentinos, existe una contribución metodológica motivada por esta misma aplicación. Específicamente, para la estimación de las demandas se propuso un modelo simple y parsimonioso, consistente con la teoría económica del consumidor, que puede ser aplicado a estudiar la demanda mundial y el posicionamiento competitivo de cualquier país en las exportaciones de un determinado sector de interés.

\section{Referencias}

Agostino, M. R., Aillo, F., \& Cardamone P. (2007). Analyzing the Impact of Trade Preferences in Gravity Models. Does Aggregation Matter? TRADEAG Working Paper 07 /4, TRADEAG - Agricultural Trade Agreements. Anderson, M., \& E. van Wincoop (2003). Gravity with Gravitas: A Solution to the Border Puzzle. American Economic Review, 93(1), 170-192.

Armington, P. S. (1969). A Theory of Demand for Products Distinguished by Place of Production. IMF Staff Papers, 16(1), 159-177.

Asche, F., \& Zhang D. (2013). Testing Structural Changes in the U.S. White sh Import Market: An Inverse Demand System Approach Agricultural and Resource. Economics Review, 42(3), 453-470. 
Baltagi, B. H. (2013). Econometric Analysis of Panel Data. 5th ed. Chichester, UK: Wiley.

Baltagi, B. H., \& P. X. Wu (1999). Unequally Spaced Panel Data Regressions with AR(1) Disturbances. Econometric Theory, 15, 814-823.

Ben Kaabia, M., \& Gil, J. M. (2007). The EU Demand for Imports of Virgin Olive Oil. 103rd EAAE Seminar 'Adding Value to the Agro-Food Supply Chain in the Future Euromediterranean Space', Barcelona, Spain.

Bengoa, M., Sanchez-Robles, B., \& Schachmurove, Y. (2015). Latin America's FDI Patterns: A Panel Data Gravity Model to Assess the Role of Regional Integration Agreements. 21 International Panel Data Conference, Central European University, Budapest.

Bhargava, A., Franzini, L., \& Narendranathan, W. (1982). Serial Correlation and the Fixed Effects Model. Review of Economic Studies, 49, 533-549.

Breusch, W. M. T., Nguyen, H. T. M., \& Kompas T. (2011). On the Fixed-Effects Vector Decomposition. Political Analysis, 19, 123-134.

Broda, C., \& Weinstein D. (2006). Globalization and the Gains from Variety. Quarterly Journal of Economics, 121, 541-585.

Comtrade (2015). International Trade Statistics Database. United Nations.

Coronel, M. N., García A. R., \& Vicentín J. (2014). Tipo de cambio real sobre la oferta de productos lácteos argentinos. Revista Científica Visión de Futuro, 18(1), 193-212.

Deaton, A., \& Muellbauer J. (1980). An Almost Ideal Demand System. The American Economic Review, 70(3), 312-326.

Depetris, G. E., Rossini, G., \& García, A. R. (2009). Competitividad del Mercosur Lácteo. Evolución en la Década Posterior a su Implementación. Santa Fe: Editorial de la Universidad Nacional del Litoral.

Depetris, G. E., Rossini G., García, A. R., \& Vicentin, M. J. (2011). Competitividad del Complejo Lácteo Santafesino. Santa Fe: UNL.

Depetris, G. E., García, A. R., \& Rossini G. (2012). Competitivi- dad de las Exportaciones Argentinas de Quesos desde la implementación del Mercosur. Revista Argentina de Lactología, 27, 9-21.

Depetris, G. E., García, A. R., \& Coronel, M. (2013). Impacto del Mercado Doméstico sobre la Oferta Exportadora de Lácteos de Santa Fe. Ciencias Agronómicas, 21(13), 27-32.

Feenstra, R. C. (1994). New Product Varieties and the Measurement of International Prices. The American Economic Review, 84(1), 157-177.

Fidrmuc, J. (2009). Foreign Languages and Trade. Working Paper 09-14, Economics and Finance Working Paper Series, Brunel University.

García, A. R., Depetris, G. E., Vicentín, J., \& G. Rossini (2013). Factores Determinantes de la Oferta Exportadora Láctea Santafesina. SaberES, 5, 71-85. 
Gohin, A., \& Féménia, F. (2009). Estimating Price Elasticities of Food Trade Functions: How Relevant is the CES-based Gravity Approach? Journal of Agricultural Economics, 60(2), 253-272.

Green, W. (2011). Fixed Effects Vector Decomposition: A Magical Solution to the Problem of Time-Invariant Variables in Fixed E ects Models? Political Analysis, 19, 135-146.

Ito, S., Chen, D. T., \& Peterson, W. F. (1990). Modeling International Trade Flows and Market Shares for Agricultural Commodities: A Modified Armington Proce- dure for Rice. Agricultural Economics, 4, 315-333.

Kee, H. L., Nicita, A., \& Olorreaga M. (2008). Import Demand Elasticities and Trade Distortions. The Review of Economics and Statistics, 90(4), 666-682.

Lema, D., Galetto, A., \& Loyato V. (2005). Estimación de la Elasticidad de la Demanda de Quesos por Segmentos y Niveles de Marcas mediante la Utilización de Datos de Scanner. Documento de Trabajo 33, IES-INTA.

Nzaku, K., Houston, J. E., \& Fonsah E. G. (2012). A Dynamic Application of the AIDS Model to Import Demand for Tropical Fresh Fruits in the USA. Conference, International Association of Agricultural Economists, Foz do Iguacu, Brazil 126721,

Pesaran, M. H., \& Zhou Q. (2016). Estimation of Time-invariant E ects in Static Panel Data Models. Econometrics Review (in press), 1-35.

Plümper, T., \& Troeger V. E. (2007). Eficient Estimation of Time-Invariant and Rarely Changing variables in Finite Sample Panel Analyses with Unit Fixed E ects. Political Analysis, 15, 124-139.

Plümper, T., \& Troeger V. E. (2011). Fixed-Effects Vector Decomposition: Properties, Reliability, and Instruments. Political Analysis, 19, 147164.

Ramirez M. A., \& Wolf C. A. (2008). Source Di erentiated Mexica Dairy Import Demand. International Food and Agribusiness Management Review, 11(1), 35-50.

Rossini, G., Depetris, G. E., \& Villanueva R. (2008). Estimación de Elasticidades de Diferentes Productos Lácteos en las Provincias de Santa Fe y Entre Ríos. Revista de Economía y Estadística, 46(1), 31-44.

Rossini, G., Vicentin M. J., García A. R., \& Coronel M. (2013). Transmisiones de Precios en el Sector Lácteo: Un Análisis del Comportamiento de los Precios de Exportación y el Recibido por los Productores. Revista FAVE. Ciencias Agrarias, 12, 1-11.

Sauquet, A., Lecocq, F., Delacote, P., Caurla, S., Barkaoui A., \& García S. (2011). Estimating Armington Elasticities for Sawnwood and Application to the French Forest Sector Model. Resource and Energy Economics, 33, 771-781. 
Tan, S. W. (2012). Structural Estimation of a Flexible Translog Gravity Model. Working Papers Series No. 1164, Department of Economics, University of Melbourne.

van Bergeijk, P. A., \& Brakman S. (2010). The Gravity Model in International Trade. New York: Cambridge University Press.

Verbeek, M. (2008). A Guide to Modern Econometrics. $3^{\text {rd }}$ Ed., London: Wiley $\&$ Sons Ltd.

Vicentin M. J., Depetris E., García A. R., \& Rossini G. (2013). Retrasos en la transmisión de precios de exportación entre los principales productos santafesinos: leche en polvo entera y quesos. Revista Ciencias Económicas, 2, 11-21.

Wan, Y., Sun C., \& Grebner D. L. (2010). Analusis of Import Demand for Wooden Beds in the U.S. Journal of Agricultural and Applied Economics, 424, 643-658.

Wooldridge, J. M. (2010). Econometric Analysis of Cross Section and Panel Data, 2nd Ed., Cambridge, Massachusetts: The MIT Press.

Yang, S-R., \& Koo, W. (1993). A Generalized Armington Trade Model: Respeci cation. Agricultural Economics, 9, 347-356.

Yang, S-R., \& Koo W. (1994). Japanese Meat Import Demand Estimation with the Source Di erentiated AIDS Model. Journal of Agricultural and Resource Economics, 19(2), 347-356.

Yilmazkuday, H. (2015). Importer-specific Elasticities of Demand: Evidence from U.S. Exports. International Review of Economics and Finance, 35, 228-234. 